\title{
Neutron diffraction studies of oxygen disorder in $\mathrm{Nd}_{2} \mathrm{NiO}_{4+d}$
}

Sumit Ranjan Maity ${ }^{1}$, Juerg Schefer ${ }^{1}$, Lukas keller $^{1}$, Monica Ceretti ${ }^{2}$, Werner Paulus ${ }^{2}$

${ }^{1}$ Laboratory For Neutron Scattering And Imaging, Paul Scherrer Institute, 5232 Villigen, Switzerland, ${ }^{2}$ Institut Charles Gerhardt, UMR 5253, CNRS-University Montpellier, 34095 Montpellier, France

E-mail: sumit.maity@psi.ch

Better understanding of oxygen diffusion in non-stoichiometric oxides becomes essential for further development of intermediate temperature solid oxide fuel cells. In this prospect, rare earth nickelates (R2NiO4+d) emerged as promising materials in which oxygen transport is driven by oxygen disorder and non-stoichiometry. Their crystal structure consists of RNiO3 perovskite layers sandwiched between RO-type rock salt layers. Extra oxygens are intercalated in tetrahedral sites inside the rock salt layer and diffusion is described by an interstitialcy mechanism [1] in which excess oxygens diffuse via apical oxygens as indicated in Fig. 1 (a). Therefore close to room temperature, oxygen diffusion is not Arrhenius-type (only at high temperature) rather a lattice activated process. In this talk, I will address structural studies which are important to get experimental evidences on oxygen migration mechanism in these oxides. I will present our single crystal neutron diffraction results on $\mathrm{Nd} 2 \mathrm{NiO} 4.25$ compound in the temperature range between 2-450 $\mathrm{K}$ and I will highlight, especially, the influence of non-stoichiometry on oxygen transport and magnetic properties in this compound.

Our neutron diffraction studies show that the average crystal structure of $\mathrm{Nd} 2 \mathrm{NiO} 4.25$ compound is monoclinic (B2/m space group) at room temperature. No structural transition has been observed in the temperature range between $2-450 \mathrm{~K}$. Reciprocal space plane mapping (on oriented single crystal) shows the evidence of 3d-ordering of excess oxygens in the whole temperature range. This result confirms the pinning of excess oxygens to the crystal lattice which makes the real structure incommensurate. However, these incommensurate oxygen superstructure reflections start to lose intensity around $400 \mathrm{~K}$ confirming the onset of oxygen diffusion through interstitials around this temperature. In our recent work [2] from scattering density studies (at room temperature) of average structure using the Maximum Entropy Method, we observed unusual high displacement factors both for equatorial and apical oxygen atoms showing large displacement amplitudes towards [001] and [110] with respect to the F-symmetry cell, respectively. This confirms the proposed interstitialcy diffusion mechanism and supports our previously obtained molecular dynamically simulated results [3] on Nd2NiO4.25 compound. Moreover, diffuse type incommensurate magnetic reflections have been observed below $100 \mathrm{~K}$ in the reciprocal space mapping. This confirms the magnetic ordering temperature for this compound which is about $115 \mathrm{~K}$ as identified from our specific heat data. Fig. 1 (b) shows the reconstructed ( $\mathrm{HOL}$ )-reciprocal plane at $2 \mathrm{~K}$ for Nd2NiO4.25. Also, we have followed temperature evolution of incommensurate magnetic reflections in between 2-100 K through elastic q-scans. No positional shifts of these magnetic reflections have been observed in this temperature range.

In conclusion, we have studied the influence of excess oxygens on crystal and magnetic structure as well as on oxygen transport for the $\mathrm{Nd} 2 \mathrm{NiO} 4.25$ compound using single crystal neutron diffraction in between 2-450 $\mathrm{K}$. We show that these excess oxygens are $3 \mathrm{~d}$-ordered below $450 \mathrm{~K}$ and are essential to have high oxygen mobility in the intermediate temperature range. Finally, the presence of diffuse-type magnetic reflections at low temperature confirms the weak inter-plane magnetic correlation among $\mathrm{Ni} 2+$ magnetic moments through oxygen interstitials.

[1] Bassat. J. M. et. al. (2013) J. Phys. Chem. C 117, 26466-26472. [2] Ceretti. M. et. al. (2015) J. Mater. Chem. A 3, 21140-21148. [3] Perrichon. A. et. al. (2015) J. Phys. Chem. C. 119, 1557-1564.

(b)
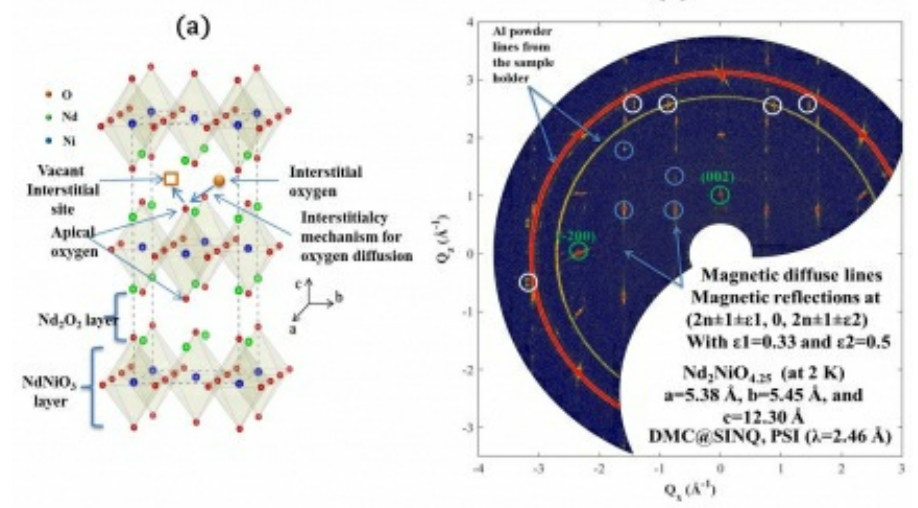
Figure 1: (a) Crystal structure of $\mathrm{Nd}_{2} \mathrm{NiO}_{423}$ at room temperature and oxygen migration mechanism is shown
with arrows and (b) a portion of the reconstructed (HOL). reciprocal plane for $\mathrm{Nd}_{2} \mathrm{NiO}_{4} .25$ measured at $2 \mathrm{~K}$ showing nuclear (green), oxygen superstructure (white) and magnetic (blue) reflections.

Keywords: Interstitialcy mechanism, Arrhenius-type diffusion, Incommensurate magnetic order. 